\title{
Ética y filosofía de la psicología
}

\author{
JUAN MANUEL CINCUNEGUI \\ Universidad del Salvador (Argentina) \\ manucincunegui@gmail.com
}

\begin{abstract}
Resumen
Este artículo se enmarca en el contexto del actual debate entre los representantes de las disciplinas de la filosofía de la mente y las ciencias cognitivas, por un lado, y los fenomenólogos, por el otro, en torno al estatuto de la consciencia y la naturaleza de la acción. A partir de la recuperación de la obra crítica del filósofo canadiense Charles Taylor, quien en The Explanation of Behaviour (1964) se enfrentó a los presupuestos del conductismo psicológico, cuyos presupuestos modificados aún están presentes en buena medida en las doctrinas cognitivistas, se ofrecen argumentos (1) contra la pretensión reduccionista de explicar lo humano a partir de lo no humano, sin por ello minimizar la importancia de las investigaciones mecanicistas, pero atentos al carácter ineludiblemente teleológico de la acción; y (2) contra el olvido insistente en lo que respecta al estatuto de los animales no humanos y el reconocimiento de la necesidad de una demarcación más fluida entre la existencia humana, animal no humana e inanimada.
\end{abstract}

Palabras claves: conductismo, teleología, acción, filosofía de la psicología, ética.

\section{Ethics and Philosophy of psychology}

\begin{abstract}
This article is part of the current debate between representatives from the disciplines of philosophy of mind and cognitive sciences on one hand, and the phenomenologists, on the other, about the status of consciousness and the nature of action. The recovery of the critical work of Canadian philosopher Charles Taylor, who in The Explanation of Behaviour (1964) faced the behaviorist challenge to humanism, and whose principles are still largely present in the cognitivists doctrines, allows us to argue against the reductionist claim that the buman can be explain through the nonbuman, without downplaying the importance of the mechanistic research in the relevant areas, but attentive to the inescapably teleological nature of action. And against the insistent forgetfulness in regard to the status of animals and nonbuman animal which means to recognize the need for a more fluid demarcation between the human, the non-buman and the inanimate.
\end{abstract}

Key words: behaviorism, teleology, action, philosophy of psychology, ethics.

Licenciado y Doctor en Filosofía. Universitat Ramon Llull (Barcelona). Docente e Investigador. Publicaciones recientes: "Charles Taylor y Alasdair MacIntyre: sobre la identidad natural" (2009); "Ecología y Habla" (2011); "The Biological Roots of Human Identity: Alasdair MacIntyre on Human and Non-Human animals" (2013); y "Las preguntas en torno al anthropos moderno" (2013). 


\section{Introducción}

Una de las cuestiones más acuciantes que se desprende de los debates en torno a la naturaleza de la mente y su funcionamiento, por un lado, y la naturaleza de la experiencia, que son los dos objetos de estudio respectivamente de (1) la filosofía de la mente y las ciencias cognitivas, y (2) la fenomenología, es la cuestión ética. Desde nuestra perspectiva, es imprescindible articular una filosofía de la psicología como presupuesto para la articulación de una ética y una filosofía social y política. En lo que a nosotros nos concierne, lo que debemos hacer es enfrentarnos a las teorías naturalistas de la mente que pretenden explicar lo humano a partir de lo no humano. Es decir, a partir de elementos comunes a la naturaleza inanimada, o a partir de la naturaleza animada no humana.

Lo que pretendemos en este artículo es presentar una serie de argumentos que nos servirán para echar luz sobre la conexión intrínseca entre: (1) el modo en el que nos entendemos a nosotros mismos, y (2) el modo por medio del cual nos orientamos hacia el bien (hacia aquello que consideramos como bueno, valioso, aquello que anhelamos o aspiramos convertirnos).

Una ética de este tipo quiere evitar dos concepciones de la moral que están muy extendidas en nuestro imaginario. Por un lado, una moral formalista como la kantiana. En este caso, lo ético se define en función del deber ser en tanto universalizable. Se trata de una ética procedimental. Por el otro lado, la ética utilitarista, que define lo ético en función de las consecuencias de las acciones, en función de lo conveniente o inconveniente para uno mismo o para el mayor número. Estas éticas están llenas de problemas. Son muy diferentes una de otra, pero comparten la convicción de que es preciso sacar del ámbito de la ética un conjunto de cuestiones que no tienen que ver con el poder humano o el florecimiento humano, como son las cuestiones de lo que creemos que es valioso en vista a nuestra dimensión finita y las preguntas en torno a la trascendencia.

Como dijimos, nosotros creemos que, para dar respuesta adecuada a las preguntas en torno a la filosofía práctica (ética, social y política) se precisa una formulación apropiada en el ámbito de la filosofía de la psicología. Para ello, dirigiremos nuestra atención a la crítica al conductismo en psicología. La razón para volver al conductismo psicológico ya pasado de moda, es que sus presupuestos, pese a las correcciones, aún están vigentes en gran medida en la psicología cognitivista, en la filosofía de la mente y otras ciencias cognitivas. 


\section{La explicación del comportamiento}

La crítica del filósofo Charles Taylor en The explanation of Behaviour (1964) al análisis del conductismo se despliega sobre la base de sus críticas a las posiciones cartesiana y empirista sobre la acción y la intención. El núcleo de su argumentación gira en torno a la convicción de que el propósito de las teorías naturalistas que subyacen a la psicología conductista consiste en ofrecer una descripción del comportamiento humano que pueda excluir toda referencia a las explicaciones teleológicas. Eso implica una visión que hace caso omiso de los principios distintivos del comportamiento de los organismos animados y otros procesos de la naturaleza. Dice Taylor (1964: 42-43):

Por ello, las leyes básicas de estas explicaciones no teleológicas no serán aquellas que gobiernan la acción, y por ello estaremos tentados a negar cualquier comportamiento que caiga bajo el título de acción. Muchos temen o esperan que el progreso gradual de la explicación científica del comportamiento llevará a nuestras acciones paulatinamente bajo leyes de este tipo, con las consecuencias de que, una por una, serán privadas de su estatuto y relegadas a la categoría de no acción. Junto a esto - y ésta es a menudo la principal razón de interés en este tema - el área en la cual podemos atribuir responsabilidad, lidiar con elogios y culpas, o administrar premios e imponer castigos, disminuirá ininterrumpidamente - hasta que en el caso límite, no quedará nada; las cortes se cerrarán o se convertirán en institutos de ingeniería humana, el discurso moral será relegado al trastero de la historia, etc.

En resumidas cuentas, la posición de Taylor se opone a la pretensión de que las leyes que explican la ocurrencia de los eventos físicos pueda servir como base para una explicación de la intencionalidad o la significatividad, es decir, a los aspectos de la antropología filosófica que se encuentran estrechamente conectados con la ética. Por ello, de acuerdo con Taylor, no sólo las éticas teleológicas, sino también el voluntarismo sartreano se ven amenazados por el reduccionismo naturalista que ejercita la filosofía conductista de la psicología (Kerr, 2004: 87). Por lo tanto, la crítica al conductismo está motivada en última instancia por la convicción de que las explicaciones mecanicistas y antiteleológicas del comportamiento humano y animal tienen efectos profundos en la filosofía moral.

Ahora bien, desde el punto de vista negativo se trata de descartar algunas teorías conductistas y sus herederas que propagan una concepción del ser humano que bloquea los ideales del humanismo. Desde el punto de vista positivo, se trata de hacer lugar a una 
recuperación de conceptos básicos de la filosofía aristotélica, tal como la encontramos en la Ética a Nicómaco. Esto nos lleva a reconocer el carácter integrado de la filosofía de la psicología, la filosofía moral y la teoría política.

Un ejemplo de ello puede ayudar a clarificar lo que pretendemos. Pensemos en las teorías contractualistas de autores como Hobbes o Locke. Estas teorías propugnan una visión de la sociedad que la percibe como una mera agregación de individuos cuyo propósito exclusivo es el beneficio de dichos individuos. En función de esta concepción, los principios políticos fundamentales a los que se adhieren gira en torno a la atribución de ciertos derechos a los individuos, al tiempo que se le otorga una relevancia secundaria a los principios de pertenencia u obligación, el cual nos impone el deber de pertenecer a la sociedad u obedecer a la autoridad (Taylor, 1985:188).

De acuerdo con este modelo, la noción de derecho juega un papel central en la justificación de las estructuras políticas. Sin embargo, el énfasis en la primacía de los intereses individuales pasa por alto las obligaciones de los agentes en lo que concierne a la sostenibilidad de la comunidad a la que pertenecemos, a la que en buena medida debemos nuestra identidad. Lo que hace plausible el individualismo liberal es el principio de autonomía y autosuficiencia de los individuos que se apoya a su vez en la epistemología triunfante de la modernidad. De acuerdo con Taylor (1985: 210):

Está claro que sólo podemos abordar el tema si nos interrogamos acerca de la naturaleza del hombre. Pero está claro que los dos extremos no se encuentran en las mismas condiciones en relación a estas cuestiones. Los atomistas están instalados con más comodidad en las intuiciones del sentido común sobre los derechos de los individuos y no muestran ningún entusiasmo ante la posibilidad de entablar esas discusiones más extensas. Y obtienen respaldo para esta actitud en las tradiciones filosóficas que llegan a nosotros desde el siglo XVII, basadas en la postulación de un sujeto sin extensión, concebido epistemológicamente como una tabula rasa y políticamente como un derechohabiente sin otra presuposición. No es un azar que estas doctrinas epistemológicas y políticas se encuentren a menudo en los escritos de las mismas figuras fundadoras.

A partir de este trasfondo, la sociedad es concebida como un compuesto de individuos desconectados, cada uno de ellos con una serie de derechos inalienables que la sociedad tiene como única función proteger. De esta manera, el atomismo se alimenta del mismo trasfondo de significaciones que sirve como fundamento a la epistemología moderna. 


\section{El caso conductista}

El éxito de la física moderna, ámbito en el cual se implementaron de la manera más exitosa las explicaciones mecanicistas en sustitución de las antiguas explicaciones teleológicas propias de las cosmologías premodernas, impulsó un mandato a todos los dominios de la investigación científica que obedecía a la convicción de que el desarrollo pleno de esos ámbitos de conocimiento estaba condicionado a la aplicación de una metodología semejante a la utilizada por la ciencia física.

En continuidad con ese mandato de la época, el psicólogo John B. Watson propuso un estudio sobre el comportamiento humano que llevara al ámbito de la psicología los principios básicos de las ciencias naturales. De acuerdo con Watson (1913), la psicología conductista debía entenderse como una rama de la ciencia natural, y su meta no era otra que la de predecir y controlar la conducta. Por esa razón, tanto la divisoria de su objeto de investigación (la conducta) entre lo humano y lo no humano, como el método introspeccionista que aborda su interpretación desde el punto de vista de la consciencia, resultaban irrelevantes.

De este modo, creció en el dominio de la psicología un movimiento que ha tenido enorme relevancia durante todo el siglo $\mathrm{XX}$, el conductismo, desarrollado posteriormente por autores como C. L. Hull y B. F. Skinner, el cual, como hemos dicho, pese a no concitar ya adhesiones entre los teóricos de la psicología, ilustra la creencia subyacente de que las leyes referentes al comportamiento humano deben tener la forma mecanicista que aun mantiene su hegemonía en amplios sectores de las ciencias humanas (Smith, 2002: 43). Del núcleo doctrinal del conductismo se desprende el propósito de remover del proceso explicativo del comportamiento humano toda referencia a la intencionalidad y la significación, y hacer lo propio con el rol de los bienes a la hora de articular la naturaleza última de los seres humanos. En la versión de Watson, el repudio del conductismo a la psicología tradicional se debía, en primer lugar, a su compromiso mentalista, en cuanto la psicología se definía a sí misma como «ciencia de los fenómenos de conciencia», y a su metodología introspeccionista (Leahey, 1998: 380).

En lo que respecta a la introspección, según Leahey (1998: 380), Watson identificó defectos sobre la base de razones empíricas, filosóficas y prácticas. Para empezar, la práctica introspectiva era incapaz de definir cuestiones básicas que su propio objeto suscitaba, como ocurría con la determinación de los atributos y la cuantificación de los estados de 
conciencia. En segundo término, a diferencia de lo que ocurría con los métodos de las ciencias naturales, el psicólogo mentalista estaba obligado a estudiar el mundo privado de la conciencia de un observador, lo cual implicaba apoyarse en un elemento personal, es decir, lo más opuesto al ideal desvinculado al que se adhería el conductista. Como ocurría con la investigación con animales, la conciencia resultaba un dato irrelevante frente a los logros obtenidos sobre la base de la observación empírica de la conducta. El investigador podía intentar una reconstrucción, a partir del comportamiento del animal, de su mente, pero esa reconstrucción, de acuerdo con Watson, era irrelevante en lo que concernía a los resultados del propio experimento. Por último, la psicología introspectivista resultaba irrelevante para la sociedad, puesto que era incapaz de ofrecer soluciones a los problemas que debían enfrentar los individuos de las sociedades modernas. Para Watson, la psicología genuinamente científica buscaba «ampliar las generalizaciones que conducían al control de la conducta humana» (Leahey, 1998: 382). Su tarea era estudiar la teoría de ajuste, en contraposición al contenido de conciencia con lo cual se pretendían alcanzar predicciones en términos de estímulo y respuesta. El anhelo de Watson era aprender los métodos generales y particulares por los cuales puede ejercitarse un control de la conducta. Lo que queda claro en lo que respecta a su metodología era que no necesitaba hacer distinción alguna entre el tratamiento que realizaba de los seres humanos y otros animales no humanos, debido justamente a la despreocupación con la cual trataba los hipotéticos «procesos conscientes».

El conductismo, en principio, se caracteriza por el hecho de que ofrece una explicación del comportamiento humano sobre la base de un modelo que toma en consideración exclusivamente las leyes de funcionamiento que rigen la relación estímulo-respuesta. Para ello, el conductista se ciñe a la observación de los movimientos burdos del organismo y su entorno, asumiendo axiomáticamente la existencia de correlaciones básicas entre dichos movimientos y operaciones cerebrales y del sistema nervioso como base material explicativa última del comportamiento ${ }^{1}$, lo cual evita la necesidad de hacer referencia a un

1 En el caso de Watson, por ejemplo, su rechazo contra la psicología mentalista e introspectivista se encuadra en una revuelta más amplia contra el pasado cultural en general. Para Watson, en consonancia con una época que promulgaba obsoleta toda raíz religiosa, la psicología tradicional estaba en continuidad con la creencia en la existencia del alma. Quienes se adherían a una concepción de la conducta a partir de un proceso centralizado en el cerebro, y no como mera respuesta a un estímulo externo, no hacían más que trasladar las funciones atribuidas en el pasado al alma, ahora a la corteza cerebral. El elemento análogo en ambas explicaciones era el hecho de ofrecer como principio explicativo último una entidad misteriosa. Como ha señalado Leahey, para 
proceso interior que subyazca a dichos movimientos. La intención es establecer una serie de leyes de correlación entre el entorno («estímulo») y el comportamiento («respuesta») prescindiendo de cualquier referencia a la intencionalidad.

Sin embargo, el conductismo no es capaz de establecer a priori que la forma de las leyes que gobiernan el comportamiento humano, y de todo comportamiento animado, sea mecanicista. Lo que subyace a su convicción es la epistemología empirista para la cual todo conocimiento genuino debe ser verificable empíricamente. Parte del problema es que, en lo que se refiere a las explicaciones teleológicas, el conductista comete una equivocación al considerar que dichas explicaciones pueden ser traducidas a las formas de las leyes mecanicistas, cuando en realidad la explicación teleológica se rige por sus propias leyes. Las explicaciones teleológicas no pueden cumplir con los requerimientos mecanicistas, entre otras cosas, porque el estado final invocado en dichas explicaciones no es análogo a las entidades que funcionan como causa antecedente en las explicaciones mecanicistas que se ciñen a la causalidad eficiente. De acuerdo con Taylor (1964: 9):

La explicación por intención involucra el uso de una explicación de forma teleológica, de explicación en términos del resultado por el cual los eventos que nos conciernen ocurren. Ahora bien cuando decimos que un evento ocurre en aras de un fin, estamos diciendo que ocurre porque es el tipo de evento que trae ese fin. Eso significa que la condición para que ese evento ocurra es que se dé un estado de cosas de tal modo que conduzca al fin en cuestión, o que tal evento es requerido para llevar a ese fin. Ofrecer una explicación teleológica de algún evento o clase de eventos, por ejemplo, el comportamiento de alguna entidad es, por lo tanto, explicarlo por medio de leyes en los términos de las cuales la ocurrencia de un evento es sostenida en dependencia del hecho de que ese evento sea requerido para cierto fin.

De este modo, la presunción empirista detrás del conductista le hace concluir que es posible realizar una reducción de la forma teleológica de la ley del comportamiento a la forma mecanicista de la misma. Sin embargo, dicha reducción es implausible. La idea de que el telos de un sistema, que se refiere a una propiedad de una totalidad integrada o irreductible, debe ser traducible a leyes explicativas es un hecho no probado. Eso nos permite sospechar que el conductista se apoya en un

Watson, ni el alma ni el córtex son entidades existentes, sino como meras estaciones que conectan estímulos y respuestas. Por ello, en lo que respecta a la predicción y control de la conducta, podemos prescindir de cualquier referencia al alma y el cerebro. 
dogma: las premisas sobre la experiencia heredadas del empirismo (Smith, 2002: 44).

En segundo término, aun cuando a priori es verdadero que las explicaciones de comportamiento en términos de intención son incompatibles con la explicación mecanicista, eso no conlleva la posibilidad de deshacernos de una ciencia del comportamiento. Lo que debemos hacer es volver a las ciencias experimentales para constatar si la mejor explicación del comportamiento es aquella que se ofrece en términos mecanicistas o teleológicos.

Sabemos que el rechazo del conductismo no se dirige únicamente a las explicaciones teleológicas en general, sino al tipo de explicación teleológica especial en la cual el telos o meta se define en término de «propósito» o «intencionalidad». La noción de acción, en su uso corriente, la explicamos habitualmente como un modo de comportamiento en el cual está involucrada la intencionalidad. Cuando hablamos de acción, en contraposición al mero comportamiento, la distinción se establece a partir de la constatación de que hay una intención o propósito detrás de dicho comportamiento que juega un rol decisivo en la producción de dicho comportamiento. Es decir, lo que diferencia a la acción del mero movimiento es la intención o deseo de un agente a partir del cual lleva a cabo un determinado comportamiento en aras de un fin. Por lo tanto, no es suficiente para decir que una acción ha tenido lugar, corroborar empíricamente un movimiento. Por otro lado, el uso corriente de términos como «logro», «intento» o «meta» sólo resulta inteligible si lo consideramos en la esfera de la acción, entendida ésta de manera distintiva frente al mero comportamiento, lo cual implica que no pueden estar sujetas dichas esferas a las mismas leyes explicativas. Desde el punto de vista del lenguaje corriente, la acción es incompatible con la determinación del comportamiento por medio de leyes mecanicistas.

Sin embargo, el análisis lingüístico no puede ofrecernos la certeza que esperábamos. La distinción lingüística entre acción y no acción demuestra el error en el que había incurrido la psicología conductista al rechazar de plano la explicación teleológica debido a la imposibilidad de ajustarse a requerimientos empiristas en detrimento de la forma de su propia ley. Pero no es suficiente para ofrecer garantías sobre el esquema conceptual adecuado para abordar la cuestión. Es necesario volverse hacia los propios fenómenos para determinar qué tipo de ley es la adecuada: las leyes teleológicas que gobiernan la acción o las mecanicistas que gobiernan el movimiento. La observación empírica muestra que las segundas no son las apropiadas para dar cuenta de los fenómenos investigados. 
De este modo, es necesario ofrecer una alternativa entre aquellos que argumentan a favor de una explicación mecanicista de la acción, y aquellos otros que niegan la posibilidad de una ciencia del comportamiento humano. Para ello es preciso investigar qué tipo de ley rige dichos comportamientos (Taylor, 1964: 48).

\section{Intencionalidad en los animales no humanos}

Una objeción al esquema esbozado nos incumbe particularmente. Se trata de los rasgos dualistas impensados en la argumentación que pretende un hipotético aislamiento metafísico del ser humano en vista a la ontología teleológica propuesta. Desde esta perspectiva, el comportamiento humano estaría gobernado por leyes de forma teleológica, mientras el resto de la naturaleza estaría gobernada por leyes de forma mecanicista. Sin embargo, la cuestión de la validez de la explicación a partir del propósito concierne lo que asemeja el comportamiento humano y no humano animal. Por lo tanto, independientemente de lo que pueda probarse en este ámbito, lo cierto es que al hablar de «acción» y «deseo» en el caso de los animales no humanos, estos difieren de la naturaleza inanimada en forma radical (Taylor, 1964: 70).

La pregunta de fondo es la siguiente: ¿Por qué razón deberíamos siquiera explorar la hipótesis de que la base de la distinción se encuentra entre los seres animados y la naturaleza inanimada en general, cuando incluso nuestra distinción entre acción y no acción se realiza sobre la base de argumentaciones que toman en consideración usos lingüísticos que son exclusivos de los seres humanos? Aunque no es posible dar una respuesta a priori a estas cuestiones, sin embargo, cabe hacer algunas consideraciones y señalar los errores de principio que han alimentado la negativa a considerar en esta cuestión a otros seres animados como agentes intencionales. Descartes, por ejemplo, creyó que los animales no humanos eran máquinas complejas. Si rechazamos estas doctrinas, sin embargo, estamos obligados a justificar una demarcación fuerte entre humanos y no humanos. En nuestro uso diario, nos referimos a los animales como agentes y portadores de deseos. Si esto es así, y no parece erróneo plantear el asunto de este modo, estamos obligados a abordar la cuestión explícitamente (Taylor, 1964: 64).

Parte del problema para determinar el estatuto de los animales no humanos surge de la indecisión en lo que concierne a la definición de la noción de la conciencia o mente, puesto que en buena medida, la atribución de acción y deseo a los animales depende del hecho de que les 
atribuyamos o no una conciencia. Si la definición de la conciencia se realiza en términos tales que esta resulta en «conciencia humana», el resultado es que los animales no pueden ser considerados agentes en los términos que nos conciernen. Esto ocurre cuando pensamos en la conciencia, como hemos visto, como algo separado y anterior a la propia acción, lo cual, en principio no permitiría estas atribuciones a los animales. Dice Taylor (1964: 65):

Los seres humanos como usuarios de lenguaje pueden devenir conscientes de los fines que desean perseguir a través del reconocimiento anterior al inicio de cualquier acción dirigida a dichos fines. Pero una conciencia de este tipo no puede atribuirse a quienes no son usuarios de lenguaje, puesto que, de aquellos que no pueden dar testimonio a otros, no podemos aseverar que puedan dárselo a ellos mismos. Por tanto los animales están excluidos por definición.

Sin embargo, si pensamos en el modo en que un animal huye frente a determinada circunstancia, cabe preguntarse cómo descubrir aquello que realmente teme. Sólo podemos hacerlo a partir de la experimentación, variando las condiciones hasta dar con aquello que causa exactamente su temor. Esto es así porque se trata de seres que no pueden establecer sus intenciones mediante el lenguaje. Sin embargo, esto no justifica que todos los atributos de las acciones animales sean de la clase que se evidencian por medio de la experimentación, es decir, aquellas que pueden ser subsumidas bajo leyes de comportamiento. El hecho de que sólo podamos determinar si un comportamiento animal justifica cierta descripción por medio del descubrimiento de la correlación de la cual ese comportamiento es una instancia, no demuestra que no podamos caracterizar su comportamiento como acción en el sentido normal. La ausencia de autotestimonialidad en los animales no implica que no podamos adscribirles acción y distinguir en su comportamiento acciones y movimientos. Por lo tanto, tenemos buenas razones para distinguir, por ejemplo, la salivación de un perro y la persecución que hace un ratón. De manera semejante, parece apropiado atribuirles deseo, frustración, dolor, miedo, etc. Es decir, existen buenas razones para considerarlos agentes. Lo cual implica que podemos clasificarlos como seres que dirigen su comportamiento. Por lo tanto, cuando hablamos de los animales no humanos, especialmente cuando se trata de los animales no humanos superiores, sus acciones no pueden ser consideradas sin remitirnos al propósito que las dirige (Taylor, 1964: 6667). 
En breve, la ausencia de la capacidad lingüística que nos permite adscribirnos estados mentales a nosotros, los humanos, no es criterio para determinar el estatuto de agencia. Eso no significa que no podamos reconocer la enorme diferencia que conlleva la capacidad que tienen los individuos humanos para reconocer sus estados de consciencia y testimoniarlos. En el caso de los animales, sólo podemos dar cuenta de su reacción a través de la experimentación en diversas condiciones, mientras que a un ser humano podemos interrogarlo. Sin embargo, eso no sólo significa que tengamos un acceso directo en el segundo caso que se encuentra vedado en el caso del animal, sino también, que el ser humano es consciente de lo que le ocurre de un modo diferente al que pueda tener el animal no humano. Para un usuario de lenguaje, pensar algo bajo una descripción X implica aplicar esta descripción a la misma. Ahora bien, es una característica de nuestro lenguaje que, en la aplicación de una descripción o concepto a algo, estamos describiendo o conceptualizando algo a lo que pueden aplicarse otras descripciones o conceptos. Un ser humano es capaz de comprender esto. Por lo tanto, «para un usuario de lenguaje la expresión 'pensar en algo como una $\mathrm{X}$ ' tiene una fuerza específica, porque es verdad que también podría pensar (lo que ellos reconocen como) la misma cosa bajo otra descripción» (Taylor, 1964: 68).

Como sostiene Taylor, del animal no podemos decir que sea consciente de aquello que para él es la misma cosa, bajo dos descripciones diferentes, simultáneamente o en ocasiones diversas. El único tipo de conciencia de los objetos que podemos atribuir a los animales es la conciencia de la relevancia inmediata de los objetos para su comportamiento, lo que Merleau-Ponty llama «valor funcional». Por lo tanto, «la única conciencia de su acción, de las metas que persigue, que podemos atribuir a los animales es aquella que acompaña la propia acción, el intento por lograr la meta» (Taylor, 1964: 68). Sin embargo, aunque esto lo diferencia de lo humano debido a que no contiene análogo al pensamiento verbal humano, se asemeja a éste en lo que respecta al hecho de que el comportamiento animal está dirigido en sentido fuerte.

No debería resultar difícil dar cuenta de este tipo de conciencia animal si consideramos la amplia gama de acciones humanas rutinarias que pertenecen al mismo nivel de conciencia. Por lo pronto, no parece adecuado decidir negativamente a priori. La respuesta debe ser el resultado de una investigación empírica respecto a qué explicación es más adecuada para dar cuenta del comportamiento animal.

Aun así, Taylor nos recuerda que hablar de intencionalidad en este caso, en consonancia con el sentido que tiene esta noción en autores 
como Merleau-Ponty, no significa equipararla a lo que Taylor llama «descripción intencional»:

Cuando hablamos de una 'descripción intencional' de algo, una acción, o un objeto, en el caso de los seres humanos, nos referimos habitualmente a la 'descripción privilegiada', por ejemplo, en cuál de las diversas descripciones el objeto es deseado o temido, o qué descripción de la acción incorpora nuestras razones para ello. Pero no podemos hablar de 'descripción privilegiada' en el caso de los animales. Y esto es una diferencia crucial en cualquier ciencia del comportamiento (Taylor, 1964: 69).

Sólo de los seres humanos podemos decir que son conscientes de sus razones para actuar, en cuanto pueden describirse a sí mismos y a otros, dichas razones. A diferencia de las especies animales cuyos comportamientos pueden ser descritos sobre la base de leyes específicas que operan de manera monótona en todos los miembros de la especie de una generación a otra, los seres humanos muestran una enorme diversidad de una sociedad a otra respecto a sus fines. Las ideas fundamentales son transmitidas por medio del lenguaje y la costumbre y alteradas en cada generación, lo que implica que el comportamiento humano es en parte una función histórica. En este sentido, sólo de los seres humanos podemos decir que tienen historia.

Por lo tanto, si nos centramos en la distinción entre los seres humanos y otras entidades no humanas, la línea de demarcación resulta más clara que aquella que distingue seres animados e inanimados. Las dificultades de esta última distinción son múltiples. Entre otras cosas, cuando nos aproximamos a los niveles inferiores de la escala filogenética nociones como «acción» o «deseo», resultan cada vez menos apropiadas (Taylor, 1964: 70). Sin embargo, lo que cuenta en última instancia es el tipo de explicación que resulta adecuada, al menos para cierto tipo de animales, particularmente el hombre y los animales no humanos superiores. Si una explicación intencional es la apropiada, será necesario dar cuenta de la evolución de esta clase de ser a través de diversos estadios que han dejado una huella filogenética en la que no existen rupturas definitivas. Dice Taylor (1964: 71):

Es posible que se pueda encontrar una progresión continua que lleve desde los sistemas inanimados no teleológicos, a través de sistemas teleológicos animados, hasta aquellos que no sólo son teleológicos sino también intencionales en carácter. Por lo tanto, podría ser que aquellas especies inferiores para las cuales el concepto 'acción' no tienen aplicación (además de aquellos subsistemas relativamente integrados en organismos superiores 
que son estudiados por los biólogos) sean de todas maneras de tal modo que su comportamiento sólo pueda ser explicado por medio de leyes teleológicas, aunque no intencionales.

Existe un conjunto de cuestiones en torno a la continuidad entre los estadios prelingüísticos y los estadios lingüísticos en el propio ser humano cuya explicación sólo resulta plausible tomando en consideración las investigaciones empíricas llevadas a cabo por aquellos que han ahondado en la comunicación con otras especies animales no humanas. Como ha señalado Alasdair MacIntyre, existe en la tradición filosófica occidental una interesada negligencia en lo que respecta al «estudio de las semejanzas y analogías de las percepciones, los sentimientos y las acciones inteligentes de ciertas especies no humanas» (MacIntyre, 1999: 13), y en el hecho de que dicho estudio podría servir para entender mejor la percepción, los sentimientos y la inteligencia práctica del ser humano. Las razones que MacIntyre expone son, en cierto sentido, análogas a las expuestas por Taylor, y como éste hace un llamado a evitar una única línea divisoria rotunda entre el conjunto de los animales no humanos y el ser humano, y un tratamiento empírico de la cuestión que preste especial atención a las especies concretas de animales no humanos cuyas características pudieran ser de particular importancia.

\section{Notas conclusivas}

En el contexto de la antropología filosófica de Taylor, The explanation of Behaviour puede leerse como un estudio sistemático a fin de recuperar el fenómeno de la subjetividad encarnada (Smith, 2002: 50). En contraposición a lo que ocurre con la explicación conductista, Taylor se alinea con aquellos que conciben al agente como un sujeto encarnado, involucrado en una esfera de significación, habilitado para actuar y estructurar a partir de dicha acción el mundo que habita. Para el conductista no existen sujetos encarnados, ni campos de significación, sólo correlaciones de estimulo-respuesta, y por lo tanto, un universo en el cual las acciones han sido reducidas a mero movimiento. La objeción de Taylor es empírica: la explicación conductista resulta inadecuada para dar cuenta de la subjetividad encarnada, debido a su compromiso con la epistemología reduccionista del empirismo moderno. El conductismo comienza su argumentación sobre la base de los requerimientos que exige cierta concepción de la explicación científica, lo cual le obliga a excluir de la misma toda referencia a los sujetos encarnados (Smith, 2002). O para decirlo de otro modo, si volvemos la mirada a la definición 
de la persona humana entendida esta como animal que se autointerpreta, como animal poseedor de logos, la objeción al conductismo y al conjunto de teorías en el ámbito de las ciencias humanas cuyos trasfondos se encuentran aun embrujados por el poder explicativo de la epistemología moderna, gira en torno a la convicción de que estas teorías se despliegan sobre la base de una confusión categorial. Las explicaciones ofrecidas por el conductismo, para continuar con el ejemplo que tenemos entre manos, hacen desaparecer al agente humano como objeto de investigación. Lo mismo ocurre con buena parte de las ciencias cognitivas. Pero sería erróneo creer que esta objeción concierne a priori a los objetos y métodos de estudio de dichas ciencias. Más bien, lo que se pone en cuestión es el afán reduccionista y colonizador que motiva una expansión radicalizada de las conclusiones acotadas de sus propios objetos de investigación a otras áreas de conocimiento, hasta el punto de hacer desaparecer los objetos de estas otras áreas, como ocurre con la tan sonada «muerte» del hombre.

\section{REFERENCIAS}

-Kerr, F. (2004). The Self and the Good. Taylor's Moral Ontology. En R. Abbey (Ed.), Charles Taylor. (págs. 84-104). Cambridge: Cambridge University Press.

-Leahey, T. H. (1998). Principales corrientes en el pensamiento psicológico. Madrid: Prentice Hall.

-MacIntyre, A. (1999). Dependent Rational Animals. Why Human Beings Need the Virtues. New York: Carus.

-Smith, N. H. (2002). Charles Taylor. Meaning, Morals and Modernity. Cambridge U.K.: Polity.

-Taylor, C. (1985). Philosophy and the Human Sciences. Philosopbical Papers 2. Cambridge: Cambridge University Press.

-Taylor, C. (1964). The Explanation of Behaviour. London: Routledge \& Kegan.

-Watson, J. (1913). Psychology as the behaviorist views it. Psychological Review, 20, 158-177.

Sumario: Introducción; 1. La explicación del comportamiento; 2. El caso conductista; 3. Intencionalidad en los animales no humanos; Notas conclusivas; Referencias. 